\section{EL ACCESO DE LA MUJER A LA "ALTA CULTURA" EN LA EUROPA DEL RENACIMIENTO}

\author{
Noelia García Pérez \\ Universidad de Murcia \\ noeliagp@um.es
}

\section{WOMEN'S ACCESS TO "HIGH CULTURE" IN RENAISSANCE EUROPE}

Cómo citar este artículo/ Citation: García Pérez, N. (2013). El acceso de la mujer a la "alta cultura" en la Europa del Renacimiento. Arbor, 189(760):a020. doi: http://dx.doi.org/10.3989/ arbor.2013.760n2006

Recibido: 2 enero 2009; Aceptado: 20 febrero 2009.

RESUMEN: Este artículo analiza cómo en un contexto que no favorecía el acceso femenino a los círculos intelectuales, el patronazgo artístico permitió a las mujeres introducirse en la Alta Cultura del Renacimiento, promoviendo una perspectiva femenina que reflejara su historia, su estatus y sus propios intereses.

PALABRAS CLAVE: Alta cultura; Renacimiento; patronazgo femenino; modelos de mujer; modelos de patronazgo.

En 1975, Natalie Zemon Davis, en su ensayo "City Women and Religious Change", señalaba cómo desde finales del siglo XVI hasta el siglo XVIII, tanto en países católicos como protestantes, la mujer sufrió la impotencia provocada por una serie de cambios que, lejos de beneficiarla, le perjudicaron en todos los sentidos y ante los que nada podía hacer (Davis, 1975, 94). Las modificaciones en las leyes matrimoniales restringieron, aún más, sus libertades y su capacidad como esposa; el número de gremios femeninos disminuyó considerablemente; el papel de la mujer en el comercio de nivel medio y en la administración de patrimonios se redujo; y la diferencia de salario entre hom-
Copyright: (c) 2013 CSIC. Este es un artículo de acceso abierto distribuido bajo los términos de la licencia Creative Commons Attribution-Non Commercial (by-nc) Spain 3.0.

ABSTRACT: This article explores how in a context that did not allow women access to intellectual circles, women could exercise power through patronage. Through the role of patronesses of the arts and the culture, women were able to access to High Culture of the Renaissance, promoting a feminine perspective that reflected their history, status and interests as women.

KEYWORDS: High culture; Renaissance; female patronage; patterns of women; patterns of patronage.

bres y mujeres se incrementó. Estas realidades llevaron a Joan Kelly a plantear en su famoso ensayo "Did women have a Renaissance?", publicado en 1977, si existió un renacimiento para las mujeres. Kelly, que circunscribió la pregunta al marco italiano, sostenía que todos los progresos de la Italia renacentista, su economía protocapitalista, sus estados y su cultura humanista, actuaron para moldear a la mujer de la nobleza como un objeto estético: decorosa, casta y doblemente dependiente, de su marido y del príncipe; afirmando que, lejos de las posturas ampliamente aceptadas hasta el momento, esta etapa histórica supuso un retroceso, una pérdida de estatus para la 
mujer con respecto a la Edad Media (Kelly, 1977). Las normas jurídicas que regían la vida de las mujeres en la Edad Moderna contribuyeron a consolidar la situación de sometimiento que imperaba en otros ámbitos de la vida femenina, avanzando únicamente en la esfera de protección ante las agresiones que pudieran sufrir del exterior, especialmente las de tipo sexual ${ }^{1}$.

Años después, en 1985, David Herlihy planteó una reconsideración a la pregunta formulada por Kelly, reexaminando la postura comúnmente asumida por la mayoría de los historiadores del arte feminista según la cual, lejos de existir un renacimiento, se trató, más bien, de un retroceso frente a la posición que las mujeres habían mantenido a lo largo de la Edad Media (Herlihy, 1985). Los casos de Catalina de Siena, Juana de Arco y otras muchas carismáticas mujeres que, a su juicio, aparecían con extraordinaria frecuencia en el mundo alto medieval, le llevaron a matizar la postura de Kelly y afirmar que, al menos en un sector de la vida social y cultural, las mujeres tuvieron un renacimiento.

Lo cierto es que en un mundo repleto de restricciones, la mujer encontró en la promoción artística una posibilidad de actuación aceptada e incluso reconocida. Tal como ha señalado Margaret L. King, del mismo modo que la mayoría de las mujeres de las clases dirigentes no gobernaron, compartiendo tan sólo algunas prerrogativas de la soberanía, no pudieron participar de una forma directa en los círculos intelectuales; pero sí lograron acceder a la Alta Cultura a través del patronazgo artístico (King, 1991, 160). Donde quiera que existieran cortes como centros de riqueza y actividad artística, las mujeres inteligentes actuaban en su papel de patronas de las artes y la cultura, como ocurre en los casos de Isabel I, Margarita de Austria, María de Hungría, Catalina de Medicis, Leonor de Toledo, Mencía de Mendoza o Isabel Clara Eugenia, por citar tan sólo algunos ejemplos.

El carácter del patronazgo femenino desde finales del siglo XV y hasta el XVIII refleja el impacto de nuevas cuestiones socioeconómicas que afectaron al estatus de la mujer (Colh, 1996). Las oportunidades para las elites femeninas en la participación de la vida intelectual del siglo XVI fueron, en realidad, más limitadas de lo que habían sido en el siglo anterior; sin embargo, el patronazgo permitió a las mujeres explorar el potencial de las artes como una proclamación de su propia identidad y estatus, e incluso de su propio gusto (Lawrence, 1997). De este modo, a inicios del Renacimiento, ciertas mujeres comienzan a desempeñar posiciones de poder y autoridad, que llevaron a Merry Wiesner a hablar de roles propiamente femenino (Wiesner, 1986). Las mujeres se definen a sí mismas en términos de sus títulos o roles, su lugar natal o familiar, y, en algunos casos, sus propios intereses o gustos. En este sentido, la promoción artística constituía el vehículo más apropiado para mostrar su riqueza y estatus, al tiempo que se erigía en la vía de escape a una posible creatividad frustrada. Aún así, las mujeres raramente encargaban grandes proyectos artísticos de carácter público, quedando reservadas sus iniciativas bien a obras de carácter privado, como la decoración del hogar; bien a actuaciones a caballo entre la esfera privada y la pública, como era la creación de la capilla privada de la familia. Algo que, sin duda, limitaba el ejercicio de su gusto artístico y el modelo de patronazgo a desarrollar.

Ciertamente, ni todas las mujeres que emprendieron el ejercicio de la promoción artística desempeñaron el mismo "modelo de patronazgo", ni todas ellas respondían al mismo "modelo de mujer". Pero... cuáles eran estos modelos de mujer, en qué consistían los diferentes modelos de patronazgo y, lo que es más importante, qué tipo de correspondencia pudo existir entre ellos.

El modelo de mujer ideal que nos ofrece Castiglione (prudente, casta, buena, discreta, afable, de dulce conversación, honesta, graciosa, avisada, discreta.... $)^{2}$ (Castiglione, 1994) se enmarca dentro la tendencia general de la época que la considera un mero adorno sometido al beneplácito de la figura masculina ${ }^{3}$. Desde Erasmo a Vives, pasando por Antonio de Guevara, Juan de Valdés o Fray Luis de León, multitud de teóri$\cos$ y humanistas vertieron su particular concepto de mujer ideal en tratados y manuales de conducta donde dejar establecidos los preceptos básicos que una dama había de seguir (Martínez Góngora, 1999). A pesar de las discrepancias existentes entre los distintos autores, todos acertaban a coincidir en las diferentes expectativas que suscitaba cada uno de los modelos de mujer. Estos prototipos femeninos venían determinados, básicamente, por el estado civil y las obligaciones que de éste se desprendían ${ }^{4}$. Doncella, casada o viuda: distintos estadios que implicaban distintas realidades, tal y como reflejaron teóricos y humanistas, al abordar la cuestión femenina. Así, Juan Luís Vives estructuró La instrucción de la mujer cristiana en tres partes dedicadas a las vírgenes, a las casadas y a las viudas. Si a las doncellas recomendaba esmerarse en recibir la educación necesaria para cumplir sus futuras obligaciones de madre y esposa (Vives, 1995, 35-181); a las casadas las exhortaba a la castidad y el amor entrañable a su esposo (Vives, 1995, 211 y 301), apenas salir de las fronteras del hogar y centrarse en "conservar a su marido y a él sólo agradar" (Vives, 1995, 287); a las viudas persuadía para evitar un segundo matrimonio, dedicando su vida a honrar la memoria de su difunto esposo (Vives, 1995, 387).

Estos modelos femeninos participaban de una serie de obligaciones comunes como eran el recogimiento; la sumisión y obediencia a la figura masculina; la castidad, entendida ésta como virginidad o fidelidad; la modestia, tanto en el gesto como en el semblante 
(Peñafiel, 2001), la humildad y el temor de Dios. Sin embargo, a pesar de los requisitos comunes, cada uno de estos prototipos femeninos traía consigo su propia realidad socioeconómica y jurídica, así como una serie de deberes que condicionaban sus actuaciones. El estatus marital de la mujer marcaba su consideración legal y con ella su situación económica, siempre condicionada por el país de origen (Ericsson, 1993; Kettering, 1989; Kuehn, 1991; Mendelson y Crawford, 1997; Muñoz, 1991; Segura, 1997; Smith, 1994); circunstancias que tenían una repercusión directa en el ejercicio de la promoción artística.

En este sentido, la generalizada inclinación a pensar que la mujer casada tenía una dependencia absoluta de la figura masculina ha comenzado a ser cuestionada por recientes investigaciones que sitúan a las damas de la aristocracia en un lugar aventajado desde el que sería necesario reconsiderar su capacidad de actuación y sus poderosas redes de influencia (Broomhall, 2011, Hickson, 2012, Mciver, 2006, Pearson, 2008). En cualquier caso, disfrutara o no de una cierta independencia financiera y de una mayor autonomía legal, lo cierto es que su realidad económica y jurídica había de enfrentarse a los condicionamientos sociales y morales. Cuando Antonio de Guevara señalaba que "a la muger honrada no le basta que lo sea, sino que lo parezca", no expresaba más que una realidad que la privaba de la vida pública, signo seguro de inmoralidad y escándalo. El espacio privado, sinónimo de virtud, era el reservado para una dama, el lugar donde había de desempeñar sus principales funciones, resumidas por este mismo autor:

"El oficio del marido es ganar hazienda, y el de la muger allegarla y guardarla. El oficio del marido es andar fuera a buscar la vida, y el de la muger es guardar la casa. El oficio del marido es buscar dineros, y el de la muger no gastarlos. El oficio del marido es tratar con todos, y el de la muger hablar con pocos. El oficio del marido es ser entremetido, y el de la muger es ser zahareña. El oficio del marido es saber bien hablar, y el de la muger preciarse en callar. El oficio del marido es zelar la honrra, y el de la muger es preciarse de muy honrada. El oficio del marido es ser dadivoso, y el de la muger es ser guardadora. El oficio del marido es vestirse como pudiere, y el de la muger es como debe. El oficio del marido es ser señor de todo, y el de la muger es dar cuenta de todo. El oficio del marido es despachar todo lo que es de la puerta afuera, y el de la muger es dar recaudo a todo lo de dentro de la casa. Finalmente, digo que el oficio del marido es grangear la hacienda, y el de la muger gobernar la familia" (De Guevara, 1539, 384).

Guardar, callar, administrar, honrar... el cumplimiento de estas obligaciones hace difícil imaginar que una mujer empleara sus esfuerzos en otro ámbito que no fuera el hogar. Por ello, sus inicios en la promoción de obras de arte quedan circunscritos dentro de los lími- tes de la familia o la iglesia. Obras, mayoritariamente destinadas a enaltecer la memoria dinástica de sus ancestros o de su esposo, en las que la mujer no tenía más función que actuar en nombre del marido que era, en última instancia, el que decidía la conveniencia o no del encargo. Retratos familiares, sepulcros y capillas funerarias constituían, entonces, los límites de actuación de la mujer casada en el ámbito de la promoción artística.

Sin embargo, esta situación variaba considerablemente con la muerte del esposo (Levy, 2003). La viuda, considerada más casta y virtuosa que la esposa por su abstención sexual, debía cuidarse de frecuentar espacios públicos y actuar discreta y modestamente para servir de ejemplo al resto de sus congéneres. A pesar de que el modelo teórico de viuda presentaba muchas similitudes con el de casada, la figura de esta mujer se erigía como un personaje con cierto poder debido a dos motivos fundamentales: su experiencia y su libertad respecto del control masculino. Junto a estos dos factores, la independencia económica emerge como el tercer factor definitivo que alza a la viuda como un ser capaz de actuar por sí mismo (M. King, 1991, 30). En este sentido, es necesario puntualizar que, si bien es cierto que las mujeres pertenecientes a las clases media y baja veían aumentar su libertad al convertirse en cabeza de familia, de poco les servía al desaparecer su principal fuentes de ingresos. Mientras, en el caso de las damas de clase alta, la muerte del esposo las situaba en una posición aventajada con respecto a su vida anterior. No sólo era el momento de percibir las arras, lo que suponía fuertes sumas de dinero y otros bienes, sino también la disposición de una mayor libertad de actuación para administrar sus propiedades $^{5}$. De hecho, un alto porcentaje de las mujeres que desarrollaron labores de patronazgo lo hicieron una vez quedaron liberadas de las funciones maritales y maternales ${ }^{6}$. Se trataba, en la mayoría los casos, de viudas que ya habían cumplido con sus deberes de madre o que nunca habían llegado a ejercer como tales como Margarita de Austria o María de Hungría (Lawrence, 1997, 13) (figs. 1 y 2). Pero no siempre se trató de una cuestión de independencia económica o de compromisos maritales; igualmente, se da la circunstancia, aunque en menor medida, de mujeres que hubieron de esperar hasta terminar con sus responsabilidades de gobierno para poder dedicarse al patronazgo. Este es el caso de Catalina Cornaro, reina de Chipre y Señora de Asolo, quien sólo después de su abdicación del trono, se dedicaría plenamente a las labores de promoción artística (Buenger, 1980). Otras patronas como Isabel I, Catalina de Medici o Juana de Austria (figs. 3 y 4), no sólo pudieron compaginar ambas facetas, sino que emplearon el arte en aras de sus propios intereses políticos.

Doncellas, casadas, viudas; con mayores o menores recursos económicos, cierta independencia jurídica, con o sin responsabilidades de gobierno... si algo 
Figura 1. B. van Orley. Margarita de Austria. Bruselas, Museos Reales de Bellas Artes

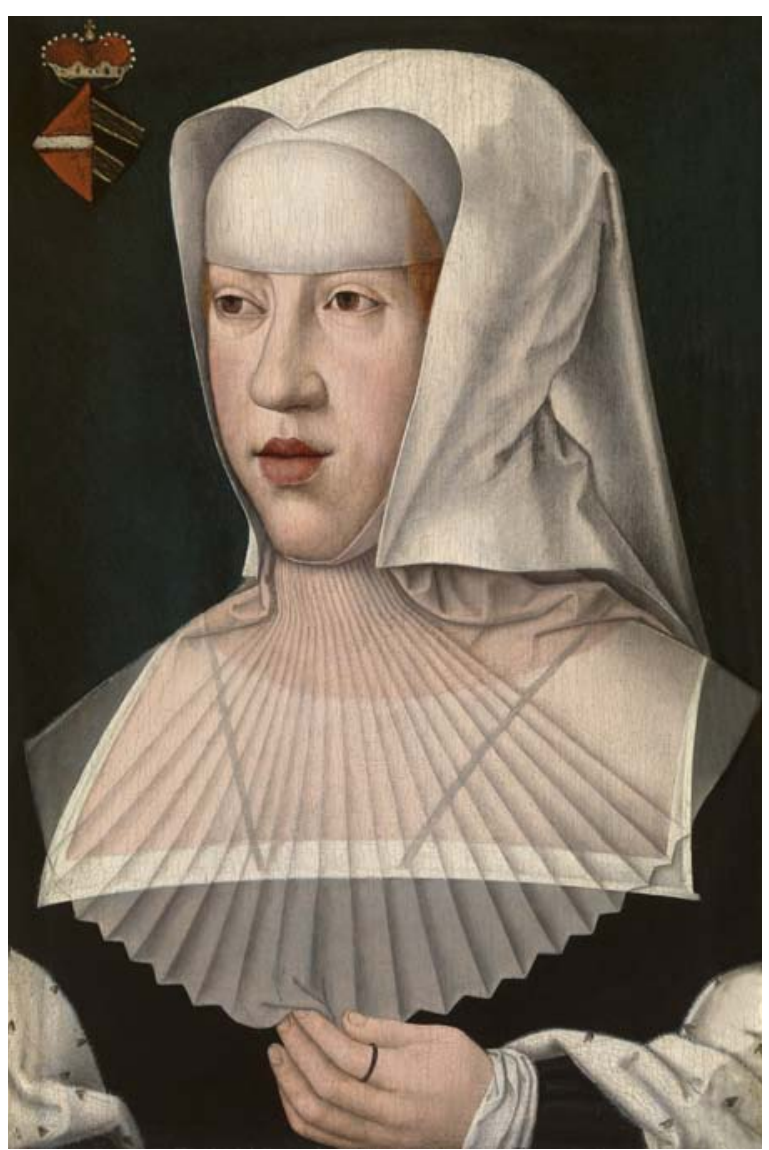

tenían en común estos modelos de mujer era el rol eminentemente pasivo que debían desempeñar en la sociedad. Como hemos apuntado, muchos investigadores consideran la esfera cultural como una de las pocas áreas donde la mujer de la Edad Moderna de buena posición económica gozó de una libertad e influencia significativa (M. King, 1991; Wiesner, 2000; Wilkins, 1975). Cierto es que la mayoría de las mujeres que desarrollaron labores de patronazgo durante su matrimonio ejercieron una labor secundaria, sometidas al beneplácito de sus cónyuges, circunstancia que ha motivado que resulte muy difícil dilucidar cuál es el verdadero promotor de muchas obras (Crum, 2001). Sin embargo, este papel fundamentalmente pasivo, obligado por las circunstancias, variaba de forma considerable en las viudas y en aquellas casadas que disponían de una independencia financiera. En este caso, la pasividad se transformaba en actividad; la esfera privada dejaba paso, prudentemente, a un plano más público (Kerber, 1988, Sharistanian, 1986); de la misma manera que las obras patrocinadas pasaban de promocionar y defender el patrimonio de la dinastía familiar y el prestigio del linaje a ser expresión de un interés propio, reservado hasta el momento a discretas manifestaciones en las obras que promocionaban? ${ }^{7}$.
Figura 2. P. Leoni y L. Leoni. María de Hungría. Madrid, Museo del Prado

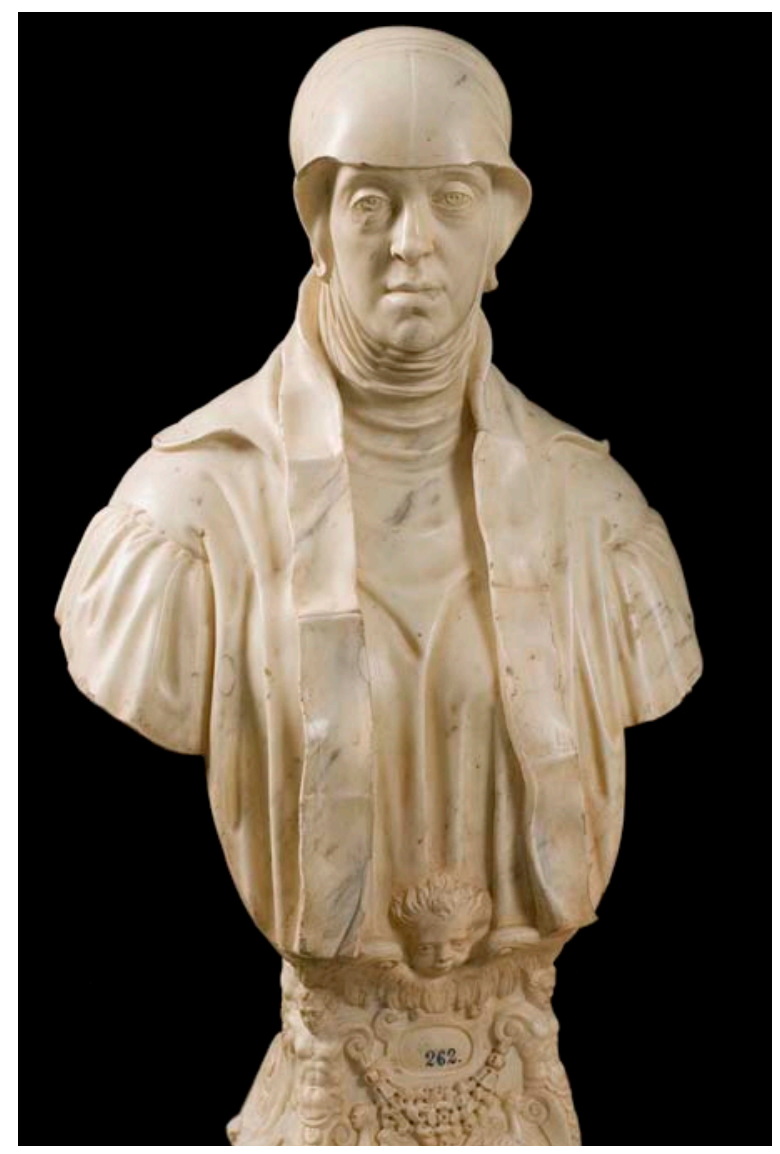

La mayoría de las mujeres que emprendieron el ejercicio de la promoción artística de manera activa comenzaron, pues, ejerciendo un patronazgo pasivo, atento a la aprobación masculina. No obstante, entre ambos extremos emerge una opción intermedia ampliamente desarrollada que denominaremos $p a-$ tronazgo activo condicionado. Si el patronazgo pasivo se caracteriza por ser aquel en el que la mujer opina, pero no decide -con el ejemplo paradigmático de Leonor de Toledo en el proceso de decoración de su oratorio personal en el Palacio Viejo de Florencia, iniciado en 1540, apenas transcurrido un año de su matrimonio con Cosme de Medici en el que la Duquesa era consultada, sin embargo, era el Duque quien tomaba las decisiones y las empleaba, en ocasiones, para extraer ventajas políticas ${ }^{8}$ (Eisenbichler, 2004, Smyth, 1997, Cox-Rearick, 1993, Eldestein, 1995 y 2001)-, el patronazgo activo implica el ejercicio del gusto personal, manifestado en la elección de un género concreto, una iconografía determinada o un estilo preciso, fruto de la experiencia y criterio femenino. Así, Catalina de Medici adoptó la imagen de Artemisa para sus retratos, dentro de unos cánones estilísticos previamente fijados (Ffolliot, 1986, 1997); Margarita de Austria escogió a Conrat Meit, como uno de los escultores favoritos (Burk, 2005) o María de Hungría 
Figura 3. Atribuido a I. Oliver. Isabel I (The Rainbow Portrait), 1600-1602. Hertfordshire, Hatfield House

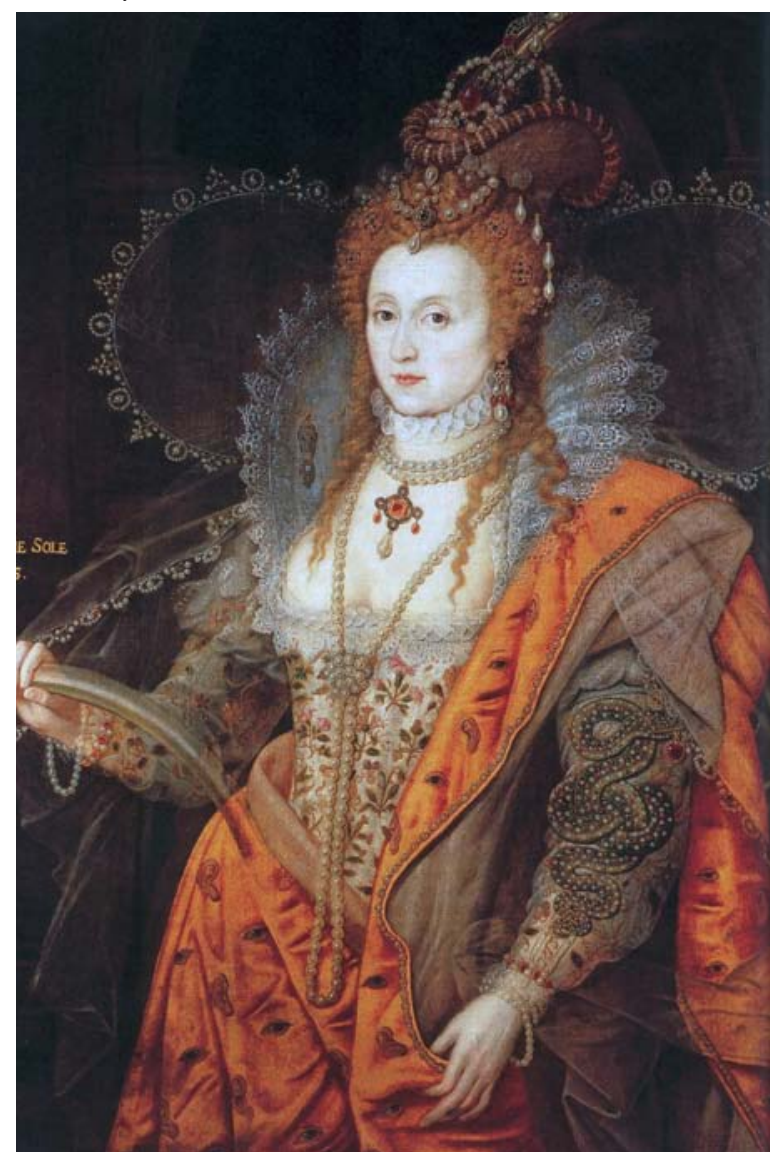

por los hermanos Leoni (Estella Marcos, 2000). Es decir, el ser activo implica tomar decisiones, elegir con cierta libertad, conforme a unos criterios personales. Sin embargo, la estética femenina actúa más o menos libremente en función del tipo de obra que comisiona y los motivos que subyacen tras el encargo. Parece, por tanto, difícil que una mujer pueda ejercer su gusto personal al comisionar un panteón familiar sin verse condicionada por el verdadero propósito que le lleva a erigir esa obra: la promoción dinástica. Sin embargo, si se tratara de su enterramiento personal, actuaría más libremente y sin otro condicionante que su propio criterio y experiencia. En el primer caso se trataría de un patronazgo activo condicionado: aqueIla actuación en la que la patrona somete su criterio personal al contexto y circunstancias del encargo y en el segundo un patronazgo activo. Uno de los ejemplos más evidentes al respecto lo encontramos en el caso de Mencía de Mendoza. La Marquesa del Zenete manifestó en su testamento su deseo de que tanto sus padres como ella fueran enterrados en la Capilla de los Tres Reyes del Convento de Predicadores de Valencia. Sin embargo, especificó cómo para sus progenitores deseaba poner "camas e bultos de alabastro" (Roest Van Limburg, 1908, 99) (fig. 5) mientras para sí,
Figura 4. A. Moro. Juana de Austria, 1560. Madrid, Museo del Prado

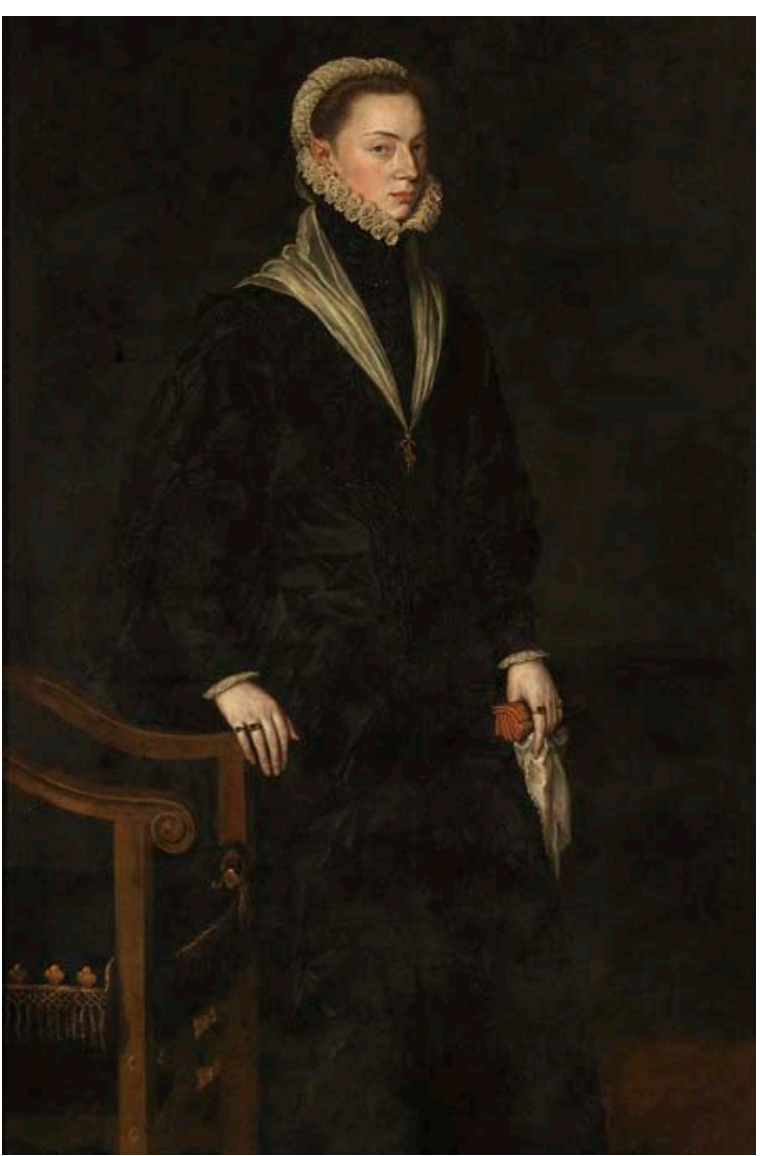

Figura 5. Cromolitografía del sepulcro de los Marqueses del Zenete, 1879. Valencia, Convento de Santo Domingo

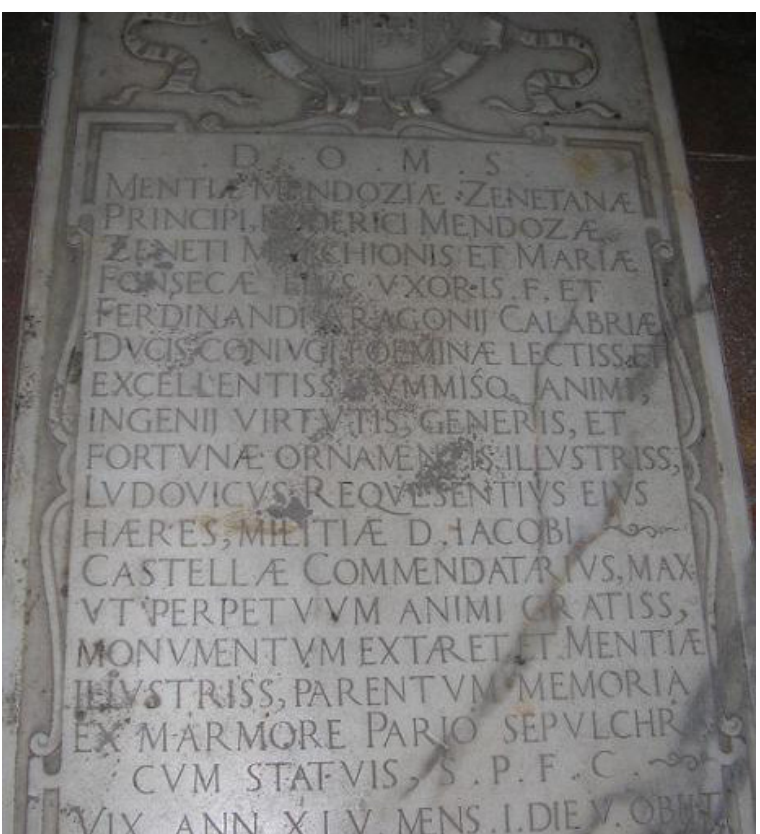

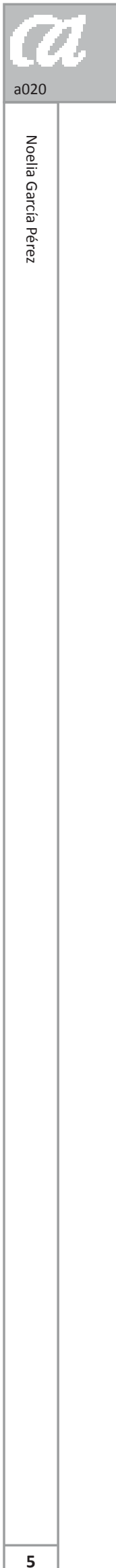


tan sólo quería que sobre su sepultura "se ponga una lancha de alabastro igual de la tierra sin otro vulto con un letrero en que se diga como mi cuerpo yace allí sepultado y se declare el dia de mi finamiento porque las personas que lo vieren y leyeren y me conoscieren tengan memoria de rogar a Dios por mi alma" (Roest van Limburg, 1908, 94-95) (fig. 6). Se trataba de dos estilos bien distintos: el primero el que sus padres hubieran deseado, el segundo el que ella misma escogía, siguiendo los parámetros de la doctrina erasmista. Ambos fueron voluntad expresa de Mencía de Mendoza, aunque la responsabilidad de la construcción recayera sobre Luis de Requesens; ambos constituyen un ejemplo de patronazgo activo, sin embargo, en el caso de la tumba de los Marqueses del Zenete asistimos a un caso de patronazgo activo condicionado, en el que el criterio personal de Mencía se ve sometido a las circunstancias particulares del encargo.

Se tratara de un patronazgo activo o pasivo, los principales proyectos que emprendieron estas mujeres perseguían unos fines muy concretos. Así, la comisión de tumbas y panteones familiares era la expresión perfecta del amor conyugal y el modo idóneo para la promoción dinástica, mientras la decoración de iglesias, capillas y conventos era considerado, bien como expresión de piedad, bien como forma de autopromoción, ya fuera personal o de su familia. No obstante, en muchos casos, las obras encargadas por patronas femeninas parecen pretender alcanzar una más alta posición personal. Tan pronto como cada una de las patronas mencionadas fue descubriendo que el arte era un instrumento al servicio del poder, un símbolo de estatus y una manera de reconocimiento social, fueron evolucionando en sus actitudes hasta convertirse en auténticas promotoras del arte, hasta el punto de llegar a rivalizar con sus cónyuges, y encontrar en el arte la vía de expresión de su propia experiencia, manifestada de numerosas formas, desde la creación de un estilo o iconografía que definiera su propio estatus o aspiraciones, al goce estético del arte, la satisfacción de la adquisición y el placer de asociarse con artistas, y más tarde, marchantes y otros coleccionistas (Lawrence, 1997).

Figura 6. Sepulcro de Mencía de Mendoza, 1554. Valencia, Convento de Santo Domingo

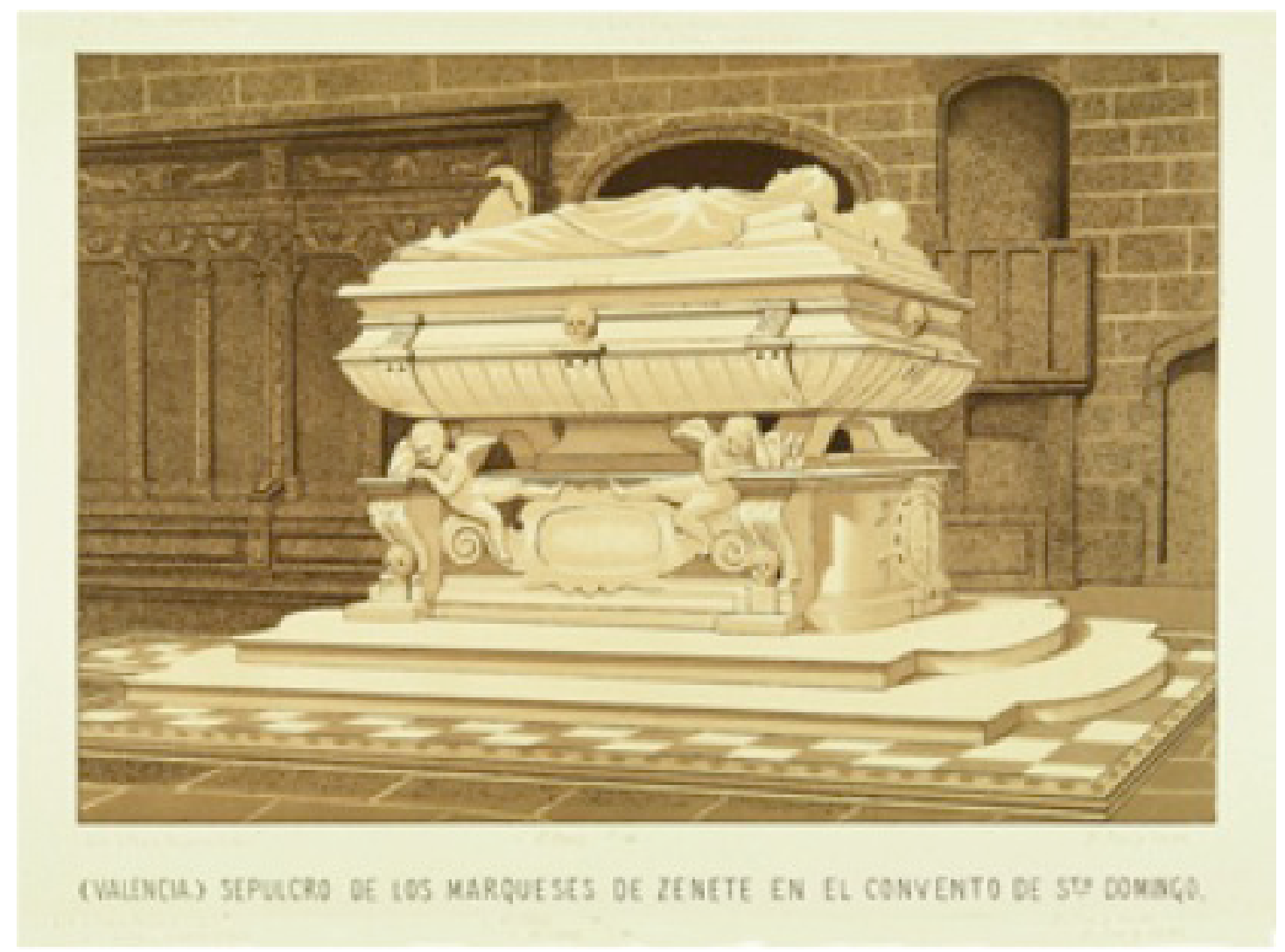


1 Sin embargo, es preciso puntualizar que la ley solamente protegía a las mujeres consideradas honradas, privilegio que recaía entre aquellas jóvenes pertenecientes a familias acomodadas y de cierto prestigio. De este modo, no se trataba de una defensa de las mujeres y de su dignidad como persona, sino de una salvaguarda de la honra familiar de las clases altas. Tal como señala Cristina Segura: "Toda mujer debe estar bajo la protección de un hombre, padre o marido, y éste es el que le proporciona la honra, que no tienen por sí mismas". (Segura Graíño, 1986, 123).

2 "Así que dexando aquellas virtudes de alma que le son a ellas comunes con el cortesano, como es la prudencia, la grandeza del ánimo, la continencia y muchas otras y asimismo aquellas calidades que se requieren en todas las mujeres, como ser buena y discreta, saber regir la hacienda del marido y la casa y los hijos, si fuere casada, y todas aquellas partes que son menester en una señora de su casa, digo que la que anda en una corte o en otro lugar donde se traten cosas de gala, paréceme que de ninguna cosa tenga tanta necesidad como de una cierta afabilidad graciosa, con la cual sepa tratar y tener correa con toda suerte de hombre honrados, teniendo con ellos una conversación dulce y honesta y conforme al tiempo y al lugar y a la calidad de aquella persona con quien hablare. (...) pero esto con tal manera de seso y de bondad lo haga, que en opinión de todos sea tan buena, prudente y bien criada, cuanto graciosa, avisada y discreta." De Castiglione, Baltasar [1531], (1994): El Cortesano, Madrid, Cátedra, p. 350.

3 Esta postura tan tradicional contrasta con la actitud más progresista, equiparable a humanistas como Erasmo, que lo lleva a defender en el tercer libro de El Cortesano la igualdad entre ambos sexos: "Y por eso, así como ninguna piedra puede ser más perfectamente piedra que otra, cuanto al ser de la piedra, ni un león más perfectamente león que otro, así un hombre no puede ser más perfectamente hombre que otro; $y$ por consiguiente no será el macho más perfecto que la hembra cuanto a la sustancia suya formal, porque entre ambos se comprende debaxo de la especie del hombre, y aquello en que el uno es diferente del otro es cosa accidental y no esencial". Ibídem, pp. 358-359.

4 Los tres estados básicos en los que se dividía la vida de una mujer quedaban determinados por su condición civil. Doncella, casada o viuda, estos fueron durante largo tiempo los prototipos femeninos básicos. Sin embargo, dentro de ellos podríamos establecer, a su vez, otros muchos modelos. Así, deberíamos considerar a la mujer dedicada a la vida religiosa, como la monja; la vinculada a los sectores marginales, como la criada, la esclava, la alcahueta o la prostituta; la ligada a otras etnias o religiones, como la morisca o la gitana; y aquella relacionada con el mundo de la magia y los poderes sobrenaturales, como la bruja. Sin embargo, exceptuando algunas de las mujeres dedicadas a la vida religiosa, los modelos de mujeres previamente citados no se abordan en este estudio por no haber desempeñado funciones de patronazgo.

5 Aunque la situación económica femenina difería sensiblemente entre los países europeos e incluso entre las diferentes ciudades-estados de un mismo país, como ocurre en el caso italiano, lo cierto es que la mujer, tras la muerte de su esposo, disfrutaba de una inusitada autonomía financiera que le permitía decidir la forma de invertir su dinero. (King, 1998, 3).

6 Según Cathering King, existían tres modelos de mujer, capacitadas legal y económicamente, para ejercer el patronazgo es estos momentos: las viudas, las hijas de personajes acaudalados que vivían en comunidades como monjas y las consortes de grandes gobernantes. Sin embargo, existe un cuarto modelo de mujer no contemplado por esta autora.
Nos referimos a aquellas mujeres cuya posición económica es independiente de su estado civil, como es el caso de Isabel I de Inglaterra, en la faceta de reina, o Mencía de Mendoza, en el ámbito de la nobleza. (C. King, 1992, 373).

7 Catherine King ha planteado la posibilidad de que las obras comisionadas por las mujeres posean cierta impronta femenina en función de las circunstancias del encargo. Así, cuando una mujer comisiona una obra para su uso personal, el trabajo resultante suele poseer su marca femenina, mientras que, si se trata de un encargo para terceras personas, normalmente hombres, como la tumba de su esposo, sólo la inscripción u otros pequeños detalles nos hará ver la participación femenina. (C. King, 1998,6).

8 No obstante, este rol pasivo, personificado en la Duquesa fue evolucionando conforme avanzaba su matrimonio, ganaba confianza y autoridad, y reconocía en el arte un instrumento de poder. De este modo, en una de las modificaciones que tuvieron lugar en la capilla entre 1545 y 1553, Leonor intervino relegando algunas de las obras que Cosme había elegido y empleado como instrumento político, y las reemplazó por imágenes más piadosas: La Anunciación de la Virgen y el Arcángel San Gabriel que vemos en la imagen. A partir de este momento, y a la vez que concluía el ciclo iconográfico de la capilla, tomó conciencia tanto de la manipulación que su esposo ejercía sobre ella, como del importante lugar ocupado por el arte en la ciudad de Florencia, donde constituía una de las máximas expresiones de poder y prestigio. Por ello, afirmó su limitado poder transformando su capilla, inicialmente concebida por su esposo como un panegírico mediceo, en una imagen de la piedad contrarreformista en consonancia con la cercanía de Leonor a la doctrina jesuítica. Fue a partir de este momento, contando ya con veintitrés años, cuando comenzó a desempeñar el patronazgo artístico de forma activa. 
Broomhall, Susan (2011): Early Modern Women in the Low Countries. Feminizing Sources and Interpretations of the Past. Aldershot, Ashgate.

Buenger Robbert, Louise (1980): "Catherina Corner, queen of Cyprus", en J. R. Brink (ed.) Female scholars. A tradition of learned women before 1800, Montreal, Eden Press Women' Publications, pp. 24-35.

Burk, Jens Ludwig (2005): "Conrat Meit. Court Sculptor to Margaret of Austria", en Women of distinction. Margaret of York. Margaret of Austria, Lovaina, Brepols, pp. 277-287.

Colh, Samuel K. Jr. (1996): "The Social History of Women in the Renaissance", en Women in the Streets. Essays on Sex and Power in Renaissance Italy, Baltimor, Londres, The John Hopkins University Press, pp. 1-15.

Cox-Rearick, Janet (1993): Bronzino's Chapel of Eleanora in the Palazzo Vecchio, Bekerley, Los Ángeles, University of California Press.

Crum, Roger J. (2001): “Controlling Women or Women Controlled? Suggestions for Gender Roles and Visual Cultures in the Italian Renaissance $\mathrm{Pa}$ lace", en Sheryl E. Reiss and David G. Wilkins (ed.), Beyond Isabella. Secular women patrons of art in Renaissance Italy, Kirksville, Missouri, Truman State University Press, pp. 37-38.

De Guevara, Antonio [1539], (1950): “Letra para Mosén Puche, valenciano, en la cual se toca largamente cómo el marido con la muger y la muger con el marido se han de haber. Es letra para dos recién casados", en Epístolas Familiares Libro Primero, Madrid, RAE, pp. 370-371.

Davis, Natalie Zemon (1975): “City Women and Religious Change", en Society and Culture in Early Modern France, Stanford, Stanford University Press, p. 94. Este artículo fue traducido al castellano en: Amelang, James S. y Nash, Mary (ed.) (1990): Historia y Género: las mujeres en la Edad Moderna y Contemporánea, Valencia, Dpto. De Historia del Arte, pp. 126-165.

Eldestein, Bruce L. (1995): Eleanora di Toledo: The Camera Verde and its Dependencias in the Palazzo Vecchio, Tesis Doctoral Inédita, Universidad de Harvard.
Eldestein, Bruce L. (2001): “Bronzino in Service of Eleanora de Toledo and Cosimo I de' Medici: Conjugal Patronage andthe Painter Courtier", en Beyond Isabella. Secular women patrons of art in Renaissance Italy, Kirksville, Missouri, Truman State University Press, pp. 225-262.

Erickson, Emy A. ERICKSON (1993), Women and property in Early Modern England, Londres, Nueva York, Routledge.

Ffolliott, Sheila (1986): "Catherine de'Medici as Artemisia: Figuring the powerful Widow", en Margaret W. Ferguson, Maureen Quilligan, and Nancy Vickers (eds.) Rewriting the Renaissance. The Discourses of sexual difference in Early Modern Europe, Chicago y Londres, Chicago University Press, pp. 227-241.

Ffolliott, Sheila (1997): "The Ideal Queenly Patron of the Renaissance. Catherine de'Medici defining herself or defined by others?", en Cynthia Lawrence (ed.) Women and Art in Early Modern Europe, Pennsylvania, The Pennsilvania State University Press, pp. 99-110.

Herlihy, David (1985): “Did Women have a Renaissance? A reconsideration", $\mathrm{Me}$ dievalia et Humanistica: Studies in Medieval \& Renaissance Culture, no13, pp. 1-23.

Hickson, Sally A. (2012): Women, Art and Architectural Patronage in Renaissance Mantua. Matrons, Mystics and monasteries, Aldershot, Ashgate.

Kelly-Gadol, Joan (1977): “Did Women Have a Renaissance?", en Renate Bridenthal y Claudia Koonz (ed.), Becoming Visible: Women in European History, Boston, Houghton Mifflin, pp. 137-163. Este artículo fue traducido al castellano en: Amelang, James S., y Nash, Mary (ed.) (1990): Historia y Género: las mujeres en la Edad Moderna y Contemporánea, Valencia, Dpto. De Historia del Arte, pp. 93-125.

Kerber, Linda (1988): “Separate Spheres, Female Worlds, Woman's Place: The Rhetoric of Women's History", Journal of America History, no 75, pp. 9-39.

Kettering, Sharon (1989), "The patronage power of early modern French noblewomen", The Historical Journal, 32, 4 , reeditado en Patronage in Sixteenth and Seventeenth-Century France, Aldershot, Ashgate, 2002, pp. 825-826.
King, Catherine (1992): "Medieval and Renaissance Matrons, Italian style", Zeitschrift für Kunstgeschichte, no 55, p. 373.

King, Catherine (1998): Renaissance Women Patrons. Wives and Widows in Italy 1300-1550, Manchester, Manchester University Press.

King, Margaret L. (1991): Mujeres renacentistas. La búsqueda de un espacio. Madrid, Alianza.

Kuehn, Thomas (1991), “Cum consenso mundualdi" Legal Guardianship of Women in Quattrocento Florence", en Law, Family, and Women: Toward Legal Antropology of Renaissance Italy, ChicagoLondres, University of Chicago Press, pp. 212-237.

Lawrence, Cynthia ed. (1997): Women and Art in Early Modern Europe. Patrons, Collectors and Connoissseurs, University Park, The Pennsylvania State University Press.

Levy, Allison (2003): Widowhood and Visual Culture in Early Modern Europe, Aldershot, Ashgate.

Marcos Estella, Margarita (2000): “El mecenazgo de la reina María de Hungría en el campo de la escultura", en Miguel Ángel Zalama y María José Redondo Cantero (coords.) Carlos V y las artes: promoción artística y familia imperial, Valladolid, Servicio de publicaciones de la Universidad de Valladolid, pp. 283-322.

Martínez-Góngora, Mar (1999): Discursos sobre la mujer en el humanismo renacentista español: los casos de Antonio de Guevara, Alfonso y Juan de Valdés y Luis de León, York (South Carolina), Spanish Literature Publications Company.

Mciver, Katherine A. (2006): Women, Art, and Architecture in Northern Italy, 1520-1580. Negoziating power, Aldershot, Ashgate.

Mendelson, Sara y Patricia Crawford (1997): Women in Early Modern England 15501720, Oxford, Nueva York, Oxford University Press, pp. 34-49.

Muñoz García, María José (1991): Limitaciones a la capacidad de obrar de la mujer casada: 1505-1975, Madrid, Universidad de Extremadura, Servicio de Publicaciones.

Pearson, Andrea (2008): Women and Portraits in Early Modern Europe. Gender, Agency, Identity, Aldershot, Ashgate. 
Peñafiel Ramón, Antonio (2001): Mujer mentalidad e identidad en la España moderna (siglo XVIII), Murcia, Servicio de Publicaciones de la Universidad de Murcia.

Roest Van Limburg, Thomas M. (1908): Een Spaansche Gravin van Nassau, Mencía de Mendoza, Markiezin van Zenete (1508-1554), Leiden, A. W. Sitjthoff's Uitgevers-Maatschappi, p. 99.

Segura Graíño, Cristina (1986): "Situación jurídica y realidad social de casadas y viudas en el medievo hispano (Andalucía)", en La condición de la mujer en la Edad Media, Actas del Coloquio celebrado en la Casa Velázquez, del 5 al 7 de noviembre de 1984, Madrid, Casa de Velázquez, Universidad Complutense, pp. 123.

Segura Graíño, Cristina (1997): "La transición del Medievo a la Modernidad", en Historia de las Mujeres en España, Madrid, Síntesis.
Sharistanian, Janet (1986): “Women's Lives in the Public and Domestic Spheres", en Gender, Ideology, and Action. Historical Perspectives on Women's Public Lives, Nueva York, Londres, Greenwood Press, pp. 1-10.

Smith, Alison A. (1994): "Locating power and influence within the provincial elite of Verona: aristocratic wives and widows", Renaissance Studies, no 8, pp. 439-448.

Smyth, Carolyn (1997): "An instance of feminine patronage in the Medici Court of Sixteenth-Century Florence. The Chapel of Eleanora de Toledo in the Palazzo Vecchio", en Women and Art in Early Modern Europe, Pennsylvania, The Pennsilvania State University Press, pp. 72-98.

Vives, Juan Luís [1523], (1995): La instrucción de la mujer cristiana, Madrid, Fundación Universitaria Española, Universidad Pontificia de Salamanca, pp. 35-181.
Wiesner, Merry E. (1986): “Women's defence of their public role", en Women in the Middle Ages and the Renaissan$c e$, Siracusa, Syracuse University Press, pp. 1-27.

Wiesner, Merry (2000): "Women and the creation of culture", en Women and Gender in Early Modern Europe, Cambridge, Nueva York, Cambridge University Press

Wilkins, David G. (1975): "Woman as artist and patron in the Middle Ages and the Renaissance", en The Roles and Images of Women in the Middle Ages and Renaissance, Pittsburgh, Centre for Medieval and Renaissance Studies, pp. 107-131. 